\title{
Raman Spectroscopic Study of Muscles Related Disorders using Drosophila Melanogaster as a Model System
}

\author{
Rekha Gautam ${ }^{\mathrm{a}}$, Upendra Nongthomba ${ }^{\mathrm{b}}$ and Siva Umapathy ${ }^{\mathrm{a}^{*}}$ \\ ${ }^{a}$ Department of Inorganic and Phsical Chemistry, Indian Institute of Science, Bangalore-560012, India. \\ *Email: umapathy@ipc.iisc.ernet.in \\ ${ }^{b}$ Department of Molecular Reproduction Development and Genetics, Indian Institute of Science, \\ Bangalore-560012, India.
}

Over the last couple of decades, biomedical Raman microspectroscopy has become an active area of research. It has been proven as one of the cutting edge technique applied in the field of histology, cytology and clinical chemistry and offers even greater promises. Raman microspectroscopy provides information on both qualitative and quantitative distribution of sample heterogeneity through the differences in the molecular structure of the biochemical components. Most importantly, it is a noninvasive technique dealing with no sample preparation and thus, avoids any possible alteration of biological sample composition. In the literature, a number of reports are present wherein they have used Raman microscpectroscopy for early diagnosis of various health disorders and to understand the underlying mechanism by constantly monitoring the chemical and morphological changes in tissue sample ${ }^{1}$. In this study, we focus on muscle related disorders using Drosophila melanogaster as a model system ${ }^{2}$.

The fruitfly, Drosophila melanogaster, has been used as an attractive model organism to understand the mechanisms underlying human diseases for years. This is because of the following reasons: shorter life span, large number of eggs, available genome database, $60 \%$ of human disease genes have counter parts in Drosophila melanogaster and conservation of developmental pathways. Muscle related diseases can be mainly divided into two groups: [1] Neuromuscular type includes Dystrophies, Spinalatrophies and Parkinson's etc and [2] Musculoskeletal and Myopathies group consisting of disorders like Cardiomyopathies, Nemalinemyopathies etc. These disorders account for overall $10 \%$ of all well known diseases in India. Thus, the objective is to monitor chemical and morphological changes during the disease progression by finding unique marker bands for every composition ${ }^{3-4}$. Monitoring the spectroscopic differences among various mutants related to muscles disorder (e.g. Troponin $\mathrm{T}(\mathrm{TnT})$ mutant $\mathrm{up}^{101}$ and $\mathrm{up}^{1}$ ) and wild type provides an insight into the cause and progression of disease at different stages in molecular level. TnT mutant affects indirect flight muscle (IFM). IFM provide a powerful genetic system to understand muscle function, structure and development. These muscles are 
physiologically similar to cardiac muscles and stucturally similar to skeletal muscles of Human.

Fig.1 presents the Raman spectra of normal and mutated adult Drosophila melanogaster's IFM recorded using Raman microspectrometer (Renishaw, RM 1000 system) with $785 \mathrm{~nm}$ laser line.

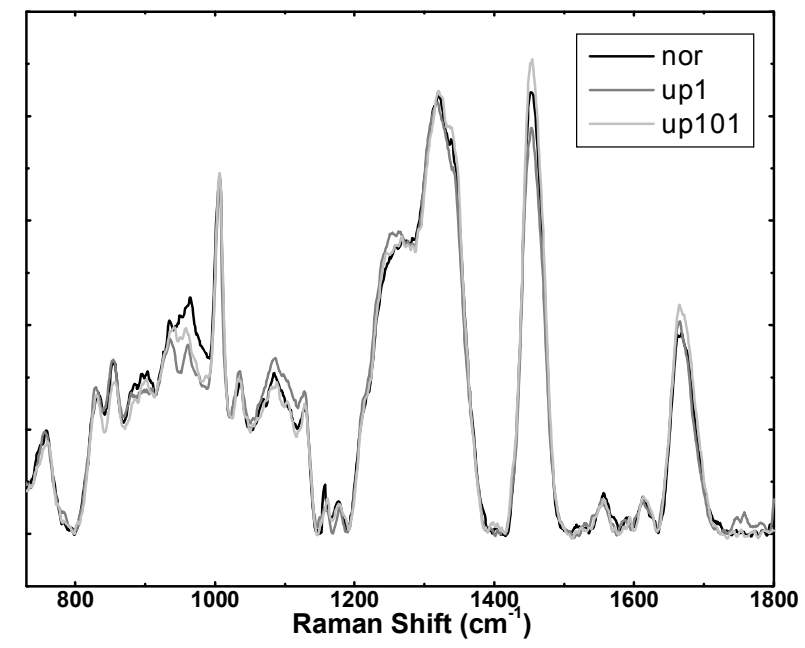

FIGURE 1. Raman spectra of normal and mutated (up ${ }^{101}$ and up ${ }^{1}$ ) adult Drosophila melanogaster's IFM.

\section{ACKNOWLEDGMENTS}

We would like to thank the Indian Institute of Science (IISc) and the Department of Science and Technology (DST) for funding this project and I would like to acknowledge Council of Scientific and Industrial Research (CSIR), India for research fellowship.

\section{REFERENCES}

1. E B Hanlon, R Manoharan, T-W Koo, K E Shafer, J T Motz, M Fitzmaurice, J R Kramer, I Itzkan, R R Dasariand M S Feld, Phys. Med. Biol. 45, R1-R59 (2000).

2. Nongthomba, U., Ansari, M., Thimmaiya, D., Stark, M. and Sparrow, J.C., Genetics, 177, $295-$ 306 (2007).

3. Ana M. Herrero, Critical Review in Food Science and Nutrition, 48, 512-523 (2008).

4. J.Louise Haston, Soren B. Engelsen, Manfred Roessle, John Clarkson, Ewan W. Blanch, Clair Baldock, Cay M. Kielty, and Timothy J. Wess, The Journal of Biological Chemistry, 278, 41189-41197 (2003). 